\title{
THE INNERVATION OF THE HAND IN RELATION TO LEPROSY
}

\author{
E. W. Price, F.R.C.S.
}

\section{Orthopaedic Surgeon to Leprosy Service, Eastern Nigeria.}

An increasing number of workers in leprosy are studying the paralysis and anaesthesia of the hands with a view to rehabilitation. It is evident that the functional loss follows definite patterns, which reflect the lesion in the nerves; but the relation between functional loss and nerve lesion is less easy to define. It is common experience that two patients may present similar clinical abnormalities of the nerve on palpation (enlargement, hardness, tenderness) while one has notable, and the other minimal, functional disability.

Studies directed to elucidate this problem are important in so far as they enable us to form a prognosis of the functional loss, and whether or not surgical intervention is likely to be of benefit. The known facts are few concerning the natural history of nerve damage and its related functional loss (with, or without, adequate treatment), and the indications for surgical intervention to replace lost function are still indistinct.

Understanding of the natural history of nerve damage in leprosy depends on careful clinical observations of the extent and progress of muscle weakness and anaesthesia, and the relation to anatomical observations at operation or on biopsy. Such deductions depend on the anatomical link between muscle (or skin) and the nerve trunk, and in investigating this, it is important to keep in mind the common variations from the normal pattern or sensory and motor nerve supply. Only thus can false deductions be avoided.

Standard works of anatomy (Gray, Cunningham, Grant) do not give details of variations in nerve supply; and this paper summarises the recognised 'anomalies of innervation' for the convenience of workers in this relatively unexplored field.

Interest in the variations of nerve supply was aroused when it was noted (in military and civil accidents) that clinical deductions from damaged hands and forearms did not always correspond with the anatomical lesions seen at operation. It has been observed, for example, that full activity of all the intrinsic muscles of the hand can exist when the median nerve is known to be completely divided (Seddon 1954). Cases are reported (Murphy 1946) where a patient with a complete division of the ulnar nerve is able to extend his fingers completely.

Sensory variations appear to be less complete than motor, but also vary within noteworthy limits.

The practical importance of these variations is that loss of muscle power or anaesthesia of a given patch of skin, may represent a lesion in a nerve, different to that which 'normally' supplies it. 
It is assumed that the investigator is aware of the various compensatory trick movements which replace loss of power in a given muscle or muscle group.

\section{Anomalous Innervation and Nerve Shunting}

The term anomalous innervation is used to describe the innervation of muscle or skin by a nerve bundle other than that described in standard text books of anatomy, and which remains associated with the parent nerve throughout its course; thus, the muscle Opponens pollicis may be supplied by a twig of the deep branch of the ulnar nerve.

The term nerve shunting is employed when the nerve bundle destined to innervate a given muscle or skin area is associated with one nerve above the elbow, but leaves that nerve below the elbow to travel down the forearm to its destination in association with another nerve. In this case, the innervation of a given structure will appear to be derived from the former nerve, if the lesion is above the elbow, or from the latter nerve if the lesion is below the elbow, e.g. at the wrist.

In view of the predilection of the leprosy lesion for the part of the ulnar nerve proximal to the elbow, and of the median nerve proximal to the wrist (Brand 1954), the important nerve-shunt in leprosy is that in which the nerve bundle leaves the ulnar nerve immediately below the elbow, and joins the median nerve in order to reach its destination; or leaves the median to join the ulnar nerve in the same region (Fig. 1). Cases of this type are described by Murphy (1946).

Shunting above the level of the upper arm is not under consideration, because leprous lesions causing clinical signs are rare above this level.

Murphy states that the only anatomical work found to describe branches between ulnar and median nerves is Poirier and Charpy's "Traite d'Anatomie Humaine", where anastomoses are described crossing from one nerve to the other between the superficial and deep layers of the flexor muscles in the upper forearm. Brash (1955) draws attention to various nerve shunts.

Therefore, the observation of weakness in a given muscle, or of anaesthesia in a given patch of skin may indicate one of the following possibilities:

(a) A lesion of the nerve which 'normally' supplies that structure.

(b) A lesion of a nerve, other than the 'normal' which is giving an anomalous supply.

(c) A lesion of a nerve bundle of the 'normal' nerve which has become involved in the lesion of a second nerve along which it is being shunted.

It must be borne in mind that continual overstretching will also cause weakness of a muscle. 


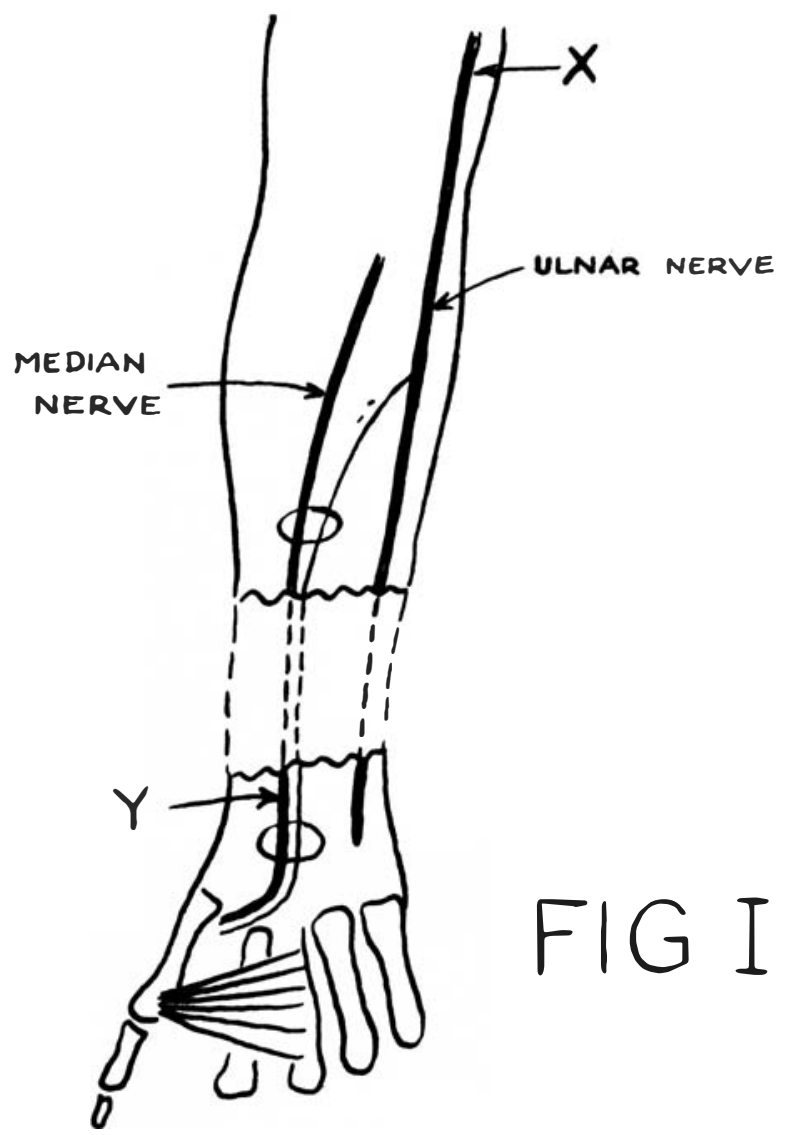

Fig. 1. Nerve shunting in the Forearm.

A nerve bundle leaves the ulnar nerve in the upper forearm to join the median nerve, which it accompanies to supply the thenar musculature. Paresis of the innervated muscle will be interpreted as due to a lesion of the ulnar nerve (if the lesion is at $X$ ) or of the median nerve (if at $Y$ ). In both places the nerve is commonly enlarged and tender in leprosy, but this abnormality does not necessarily imply any functional disability.

\section{The 'Normal' Motor Innervation of the Hand and Forearm}

If Flexor pollicis brevis is excluded, the classical innervation of the small muscles of the hand is such that the median nerve innervates the Abductor pollicis brevis, Opponens pollicis and the lateral two Lumbricals, and the ulnar nerve supplies the other intrinsic muscles of the hand. Flexor pollicis brevis is variably supplied by the ulnar nerve, or median nerve, or both (Fig. 2). This classical innervation is true in $80 \%$ of cases, but it must be realised that in a leprosarium of 1,000 patients, this means that no fewer than 400 hands will have a different innervation.

In the forearm, the ulnar nerve 'normally' supplies Flexor carpi ulnaris and the medial two bellies of Flexor digitorum profundus. 


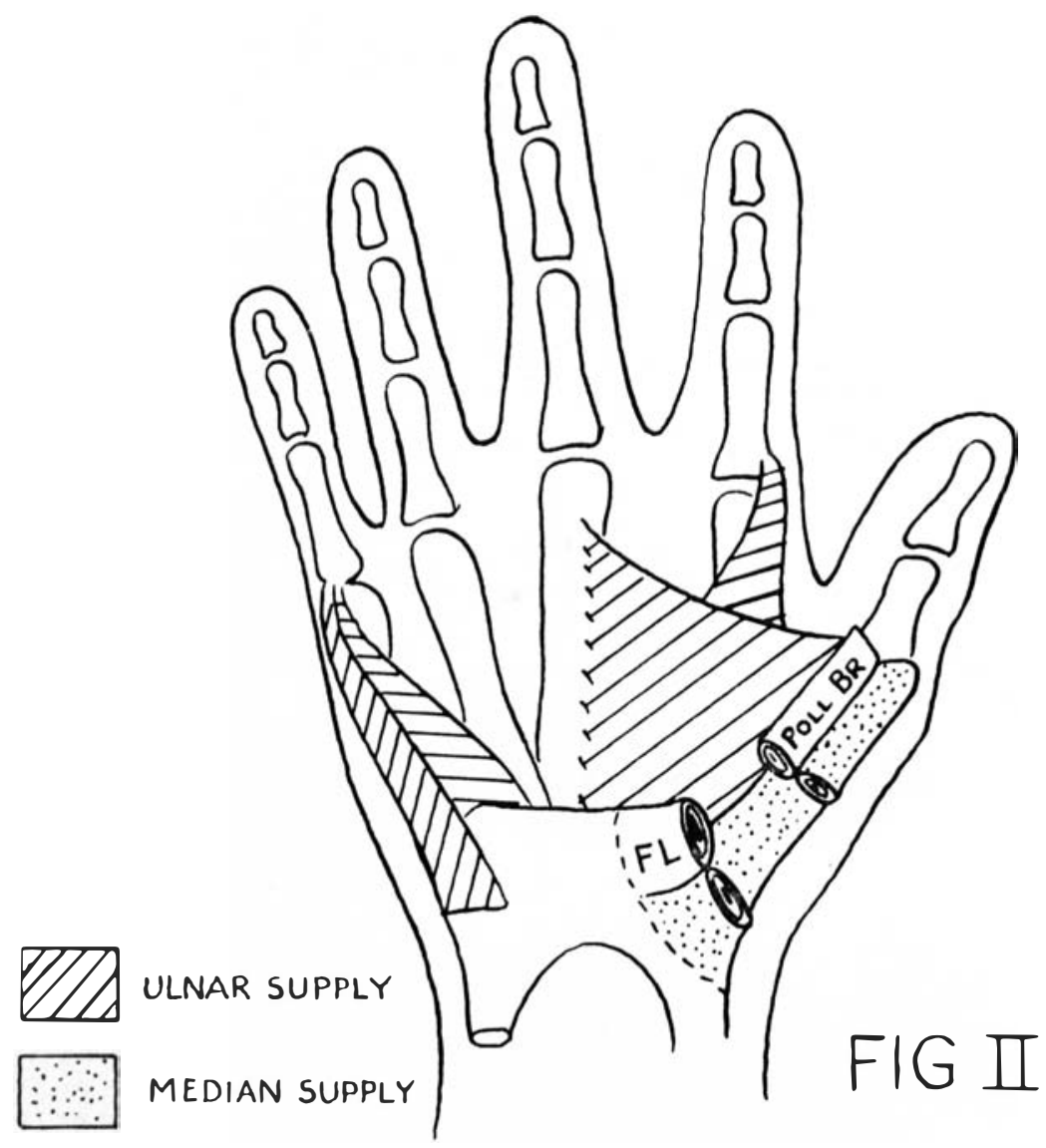

Fig. 2. The 'Normal' Innervation of the Muscles of the Hand.

Excluding Flexor Pollicis Brevis, this is the innervation on $80 \%$ of hands. Of this group, Flexor Pollicis Brevis is supplied by the ulnar nerve in two-fifths of the the cases, by the median in two-fifths, and by both in the remaining one-fifth.

\section{Anomalous Motor Innervations of Hand and Forearm}

The anomalous motor innervations of the hand range from complete ulnar innervation to complete median innervation.

In the cases reported by the Medical Research Council (Seddon 1954), the thenar group were entirely supplied by the ulnar nerve in $2 \%$ of all cases (i.e. in 40 hands in a leprosarium of 1,000 patients), and entirely by the median in $3 \%$ (60 hands). In the latter group, a third of the cases $(1 \%)$ showed median innervation of the first dorsal interosseus as well. In view of the frequency of wasting of this latter muscle in leprosy, it should be recalled that in only 20 hands in a leprosarium of 1,000 patients does wasting of this muscle represent other than an ulnar nerve lesion.

In the forearm, the innervation of Flexor digitorum profundus must be kept in mind, in view of its involvement in leprosy in one 

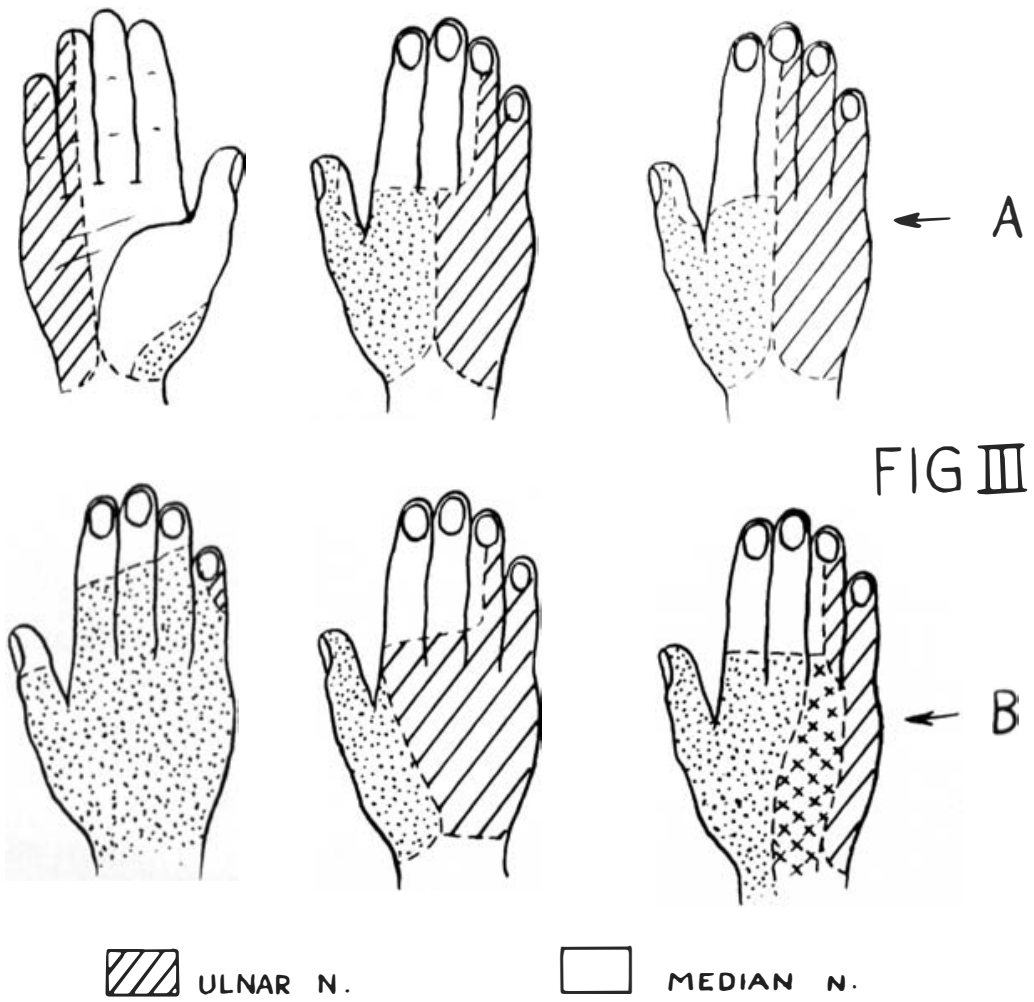

MEDIAN N.

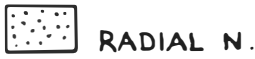

POST CUT $N$. OF FOREARM

(A) The 'normal' cutaneous innervation of the hand. Clinical experience indicates two patterns of innervation on the dorsum, as shown.

(B) Less common cutaneous innervations of the hand. It will be noted that the only area on the dorsum which is invariably supplied by the ulnar nerve is the medial half of the distal phalanx of the fifth finger; also, that the only area always supplied by the median nerve on the dorsum is the distal phalanx of the index and the lateral half of the distal phalanx of the long finger.

out of four or five cases of nerve damage in leprosy (Thomas 1954). There are several variations to the standard description, in which the portion of muscle which is motor to the fourth and fifth finger is supplied by the ulnar nerve. Ulnar innervation may extend to the belly for the long finger, especially when Flexor pollicis brevis is entirely supplied by the ulnar; but it has never been recorded as supplying the belly to the index finger. Median innervation has extended as far as the belly to the fifth finger, supplying in this case the whole muscle. Murphy claims that this is so common that it can hardly be claimed to be anomalous (see also Brash 1955).

The bellies to the third and fourth fingers often have a double median-ulnar innervation. It is evident that the only movement of Flexor digitorum profundus that can safely be taken to implicate a definite nerve is that of the index, of which weakness or loss of 
distal interphalangeal flexion certainly indicates a median nerve lesion.

\section{The 'Normal' Sensory Innervation of the Hand}

There are two accounts of the 'normal' sensory innervation of the hand. In one account (Grant 1947), the ulnar nerve supplies the skin of the palmar and dorsal surfaces of the fifth finger, and the medial half of the fourth finger. In the other (Cunningham 1931), the ulnar nerve supplies the medial one and a half fingers on their palmar surface, but the medial two and a half on their dorsal surface. It is claimed that the latter is the commoner observation in clinical cases.

\section{Anomalous Sensory Innervations of the Hand}

In view of the significance of the sensory loss as an indication of nerve damage, it is unfortunate that no systematic account exists of the variations in sensory supply. Notable variations have been observed (Fig. 3) and it will be seen that the only area on the dorsum of the hand that is consistently supplied by the ulnar nerve is the medial half of the distal phalanx of the little finger. Similarly, the only dorsal area consistently supplied by the median nerve is the distal phalanx of the index and of the long finger.

The skin of the dorsal part of the hand has been reported (Grant 1947) as innervated by the dorsal cutaneous nerve of the forearm as far as the base of the fingers in one case.

\section{Correlation of Sensory and Motor Innervation}

No studies have been found in the literature correlating motor and sensory innervation of the hand.

\section{The Return of Sensation to Denervated Skin}

It is well known that skin which has become anaesthetic in the course of leprosy may wholly or partially recover sensation under treatment. It is assumed that function is restored in nerve fibres and end-organs that have not been irreparably damaged or atrophied in the course of the disease. While this will certainly be the case where recovery is fairly rapid, it is less likely to occur in cases where the loss has lasted for a long time. In these cases, the end-organs are likely to be atrophied beyond recovery. However, the fibrils serving the pain sense are not normally associated with end-organs, and return of pain sensation can be hoped for even where other functions are lost. The gain of pain sense will be important in the protection of the part against injury.

The only studies found in the literature concerning the regeneration of nerves in denervated skin concern that of the re-innervation of free skin-grafts. This is summarised by Padgett (1948) with 
references. Although re-innervation is usually along the track of the original nerve fibrils, it has also been observed along the line of the original small blood and lymph vessels. This possibility must be kept in mind in cases of leprosy where the track of the original nerve fibrils is likely to be inhospitable to new fibrils.

The restoration of sensation will be due to one, or both, of two factors. Re-innervation or renewed function of the original nerve may be occurring; alternatively, diminution of the area of anaesthesia is possible by spread from surrounding areas where the nerves are still relatively healthy. In small areas, this latter phenomenon can completely re-innervate the skin. It is noted that renewed function due to renewal of the original nerve is likely to be observed by diminution of the area of sensory loss proximally and laterally. Spread from surrounding functioning nerves is more likely to be in progress if the gain in sensation occurs all round the area simultaneously.

\section{SUMMARY}

1. More information is urgently needed concerning the natural history of nerve-damage in leprosy, particularly under modern treatment.

2. Careful observation of loss of muscle power and skin sensation must take into consideration the recognised variations in nerve supply.

3. The commonly recognised variations of muscle and skin innervation in the hand are summarised.

I am indebted to the Director of Medical Services, Eastern Nigeria, for permission to publish this paper.

\section{REFERENCES}

1. BRAND, P. W. (1954) "The place of Physical Medicine and Orthopaedic Surgery in Leprosy". Leprosy Review 25, 5.

2. BraSH, J. C. (1955) Neurovascular Hila of Limb Muscles. London (E. \& S. Livingstone).

3. CunNingham, D. J. (1931) Textbook of Anatomy 6th Edition, London (Oxford University Press).

4. Grant, J. C. B. (1947) An Atlas of Anatomy. London (Balliáre), Tinday \& Cox).

5. Gray's Anatomy (1946). 29th Edition, London (Longmans, Green \& Co.)

6. HigheT, W. B. (1943) "Innervation and Function of the Thenar Muscles". Lancet, 1, 227.

7. MurPhy, F., et al. (1946) "Anomalous Innervation of the Intrinsic Muscles of the Hand". Surgery, Gyne., and Obstet.1946, 15.

8. Padgett and Stephenson. (1948) Plastic and Reconstructive Surgery. New York (C. C. Thomas).

9. RowntreE, T. (1949) "Anomalous Innervation of the Hand Muscles." Journ. of Bone and Joint Surg. 31, 505.

10. Seddon, H. J. (1945) Ed. Peripheral Nerve Injuries Medical Research Council, London. (H.M. Stationery Office).

11. Thomas, R. E. (1954). "An Investigation into Paralysis Patterns in the Forearm and Hand in Leprosy." Leprosy Review, 25, 2. 